\title{
Concavity of the Feasible Signal-to-Noise Ratio Region in Power Control Problems
}

\author{
Wing Shing Wong, Fellow, IEEE
}

\begin{abstract}
Signal-to-noise (SNR) ratio is commonly regarded as a reliable performance measure for wireless communication systems. Knowledge of fundamental properties of the feasible SNR region can facilitate the performance optimization of multi-user wireless systems. This paper examines the concavity of the feasible SNR region. In particular, it is shown that for systems with only three users, the feasible SNR region is always concave. As concavity for 2-D systems is well known and concavity for 4-D systems does not hold in general, this result fills in a gap on this issue. A concavity result for systems with a general number of users is also established under certain technical conditions.
\end{abstract}

Index Terms-Concavity set, feasible signal-to-noise ratio (SNR) region, power control.

\section{INTRODUCTION}

$\mathbf{S}$ IGNAL-TO-NOISE ratios (SNRs) are key performance indicators for wireless communication systems. The SNR of a user is defined by dividing the power of the received signal by the sum of the power from all noises, including interfering transmissions from other users and thermal noises. These ratios are typically analyzed under the assumption that interfering noises form Gaussian processes. Although there are limitations of this assumption, as reported in Sunay and McLane [1] and Chen and Wong [2], the importance of these ratios as a performance measure for wireless systems has never been doubted.

For a wireless system with $N$ users, the SNRs form an $N$-D vector. In this paper, all users are assumed to be transmitting with strictly positive power; hence, all the ratios are strictly positive and the SNR vector is a point lying in the positive orthant, $\mathbb{R}_{+}^{N}=\left\{\left(x_{1}, x_{2}, \ldots, x_{N}\right) \in \mathbb{R}^{N}: x_{i}>0, \forall i\right\}$. The feasible region defined by the SNR vectors is the focus of this study. For the case where there are only two users, the feasible region can be easily described as the region in $\mathbb{R}_{+}^{2}$ bounded by the two axes and the curve

$$
\left\{\left(a x, \frac{b}{x}\right): x \in \mathbb{R}_{+}\right\}
$$

for some strictly positive $a$ and $b$ whose values are dependent on the channel gains. This feasible region is obviously concave in the sense that its complement in $\mathbb{R}_{+}^{2}$ is convex, so that the

Manuscript received November 12, 2007; revised May 27, 2009; accepted July 01, 2010. Date of current version March 16, 2011.

The author is with the Department of Information Engineering, The Chinese University of Hong Kong, Shatin, Hong Kong (e-mail: wswong@ie.cuhk.edu. hk).

Communicated by H. Boche, Associate Editor for Communications.

Digital Object Identifier 10.1109/TIT.2011.2111990 line joining any two points in the complement is contained in the complement.

It is natural to ask whether this concavity property of the feasible region holds in higher dimensional systems. This has been a longstanding open question. Sung [3] provided an interesting insight into this problem by showing that the feasible region is log-convex. Subsequent papers dealing with geometric properties of the feasibility region include Catrein et. al. [4], Boche and Stanczak [5], [6], Imhof and Mathar [7], [8], Stanczak and Boche [9]. In particular, it has been shown in [9] that the concavity result does not hold for a general four user wireless system. The situation for a general three user system remains open.

In this paper, we investigate the concavity of feasible SNR regions. Main results include the following:

1) The feasible SNR region of a general three user system is concave.

2) Consider a system with a channel gain matrix that can be represented as a product of a diagonal matrix with a symmetric matrix. If the gain matrix has only one eigenvalue strictly greater than 1 and the remaining eigenvalues strictly less than 1, the feasible SNR region is concave.

We will demonstrate by concrete examples that for systems with gain matrices not satisfying the stated eigenvalue condition the feasible SNR region is not necessarily concave. In fact, the geometry of a general feasible SNR region can be extremely complicated. For example, given any two points, $x$ and $y$, on the boundary that divides the feasible region and its complement in $\mathbb{R}_{+}^{N}$, the line:

$$
\{\alpha x+\beta y: \alpha+\beta=1,0<\alpha, \beta<1\}
$$

does not necessarily lie completely outside nor inside the feasible SNR region. We will show by numerical examples that the line can be divided into segments, some of which are outside and the remaining ones are inside of the feasible SNR region.

The main tool used in establishing these results originates from an idea proposed in a paper by Weinberger [10] in which he provided a simple proof of a theorem of Lax [11]. The essence of this idea lies in the construction of a special two-variable characteristic polynomial. However, beyond this basic contraption, there is little relation between the arguments used in [10] and this paper.

The organization of the rest of the paper is as follows. Section II presents a description of the basic model and assumptions. In Section III, the two-variable polynomial alluded to earlier is introduced and some of its fundamental properties are described. Moreover, a basic concavity result for a general number of users is established. In Section IV, concavity for a 
general 3-D system is proven. Numerical examples are provided in Sections V and VI offers some concluding remarks.

\section{BASIC MODEL AND BACKGROUND INFORMATION}

The basic model discussed in this paper consists of $N$ transmitter-receiver pairs, all sharing the same radio spectrum. Let $G_{i j} \geq 0$ represent the channel gain between the $j$-th transmitter and the $i$-th receiver. The $N \times N$ channel gain matrix, $\mathbf{G}$, is defined to be $\left(G_{i j}\right)$. (For any matrix or vector, $\mathbf{M}$, we use the notation $\mathbf{M} \geq \mathbf{0}$ to denote that all components of $\mathbf{M}$ are nonnegative, $\mathbf{M}>\mathbf{0}$ to denote that $\mathbf{M} \geq \mathbf{0}$ and $\mathbf{M} \neq \mathbf{0}$ and $\mathbf{M} \gg \mathbf{0}$ to denote that all the components are $\mathbf{M}$ strictly positive). In practice, $\mathbf{G} \gg \mathbf{0}$. However, to establish the results in this paper, it is sufficient to assume that all diagonal terms of $\mathbf{G}$ are strictly positive and the interference matrix, $\mathbf{Z}=\left(Z_{i j}\right)$, defined by

$$
\mathbf{Z}=\left(\begin{array}{cccc}
G_{11}^{-1} & 0 & \cdots & 0 \\
0 & G_{22}^{-1} & \cdots & 0 \\
\vdots & \vdots & \ddots & \vdots \\
0 & \cdots & 0 & G_{N N}^{-1}
\end{array}\right) \mathbf{G}-\mathbf{I}
$$

is primitive [12] ( $\mathbf{I}$ is the $N$ by $N$ identity matrix).

Let $\mathbf{p}=\left(p_{1}, p_{2}, \ldots, p_{m}\right) \gg \mathbf{0}$ denote a power vector and $\boldsymbol{\eta}=\left(\eta_{1}, \eta_{2}, \ldots, \eta_{m}\right) \gg \mathbf{0}$ a noise power vector. The SNR for the $i$-th user, represented by $\Gamma_{i}(\mathbf{p})$, is defined by

$$
\Gamma_{i}(\mathbf{p})=\frac{G_{i i} p_{i}}{\sum_{j \neq i} G_{i j} p_{j}+\eta_{i}}=\frac{p_{i}}{\sum_{j \neq i} Z_{i j} p_{j}+\frac{\eta_{i}}{G_{i i}}} .
$$

Let $\Gamma(\mathbf{p})=\left[\Gamma_{1}(\mathbf{p}), \Gamma_{2}(\mathbf{p}), \ldots, \Gamma_{N}(\mathbf{p})\right]^{T}$ represent an $N$-dimensional column SNR vector. For the discussions in this paper, there is no loss in generality working with $\mathbf{Z}$ instead of $\mathbf{G}$. More specifically, for a given interference matrix $\mathbf{Z}$ one can view $\Gamma(\mathbf{p})$ as a continuous mapping from $\mathbb{R}_{+}^{N}$ to itself and define the feasible $S N R$ region, $\mathcal{F}(\mathbf{Z})$, to be the closure of the image set $\Gamma\left(\mathbb{R}_{+}^{N}\right)$ in $\mathbb{R}_{+}^{N}$. Let $S(\mathbf{Z})$ denote the set of limit points $\lim _{c \rightarrow \infty} \Gamma(c \mathbf{p})$ for some $\mathbf{p} \in \mathbb{R}_{+}^{N} \cdot S(\mathbf{Z})$ contains elements of the form

$$
\left[\frac{p_{1}}{\sum_{j} Z_{1 j} p_{j}}, \ldots, \frac{p_{N}}{\sum_{j} Z_{N j} p_{j}}\right]
$$

for some $\left(p_{1}, p_{2}, \ldots, p_{N}\right) \gg \mathbf{0}$.

Based on previous works reported in the literature, the structure of a feasible SNR region, $\mathcal{F}(\mathbf{Z})$, can be understood in the following way [3]. For any point $\mathbf{z}$ in $S(\mathbf{Z})$, the open line segment defined by $\{c \mathbf{z}: 0<c<1\}$ is in $\Gamma\left(\mathbb{R}_{+}^{N}\right)$, but $c \mathbf{z}$ is not in the feasible region if $c>1$. It follows that $S(\mathbf{Z})$ is in the boundary set of the feasible SNR region.

Definition: An SNR feasible region, $\mathcal{F}(\mathbf{Z})$, is concave if for any two distinct points, $\boldsymbol{\xi}$ and $\Psi$, on the boundary $S(\mathbf{Z})$, the open line

$$
\{\alpha \boldsymbol{\xi}+\beta \Psi: \alpha+\beta=1,0<\alpha, \beta<1\}
$$

is outside of $\mathcal{F}(\mathbf{Z})$.
For subsequent discussions, let $\rho(\mathbf{M})$ represent the spectral radius of a matrix $\mathbf{M}$. If $\boldsymbol{\Gamma}=\left[\Gamma_{1}, \Gamma_{2}, \ldots, \Gamma_{N}\right]^{T}$ is an SNR vector, let $\mathbf{D}_{\Gamma}$ denote the corresponding diagonal matrix

$$
\left(\begin{array}{cccc}
\Gamma_{1} & 0 & \cdots & 0 \\
0 & \Gamma_{2} & \cdots & 0 \\
\vdots & \vdots & \ddots & \vdots \\
0 & \cdots & 0 & \Gamma_{N}
\end{array}\right)
$$

Under this notation, $\boldsymbol{\Gamma}$ is contained in $S(\mathbf{Z})$ if and only if there exists a power vector, $\mathrm{p} \gg \mathbf{0}$ such that

$$
\mathrm{D}_{\Gamma} \mathrm{Zp}=\mathbf{p}
$$

\section{BASIC ANALYSIS}

Consider a fixed interference matrix, $\mathbf{Z}$, with a feasible region $\mathcal{F}(\mathbf{Z})$. Let $\boldsymbol{\xi}$ and $\boldsymbol{\Psi}$ be distinct elements on the boundary $S(\mathbf{Z})$. Note that $\boldsymbol{\xi} \gg \mathbf{0}$ and $\Psi \gg \mathbf{0}$. Let $\mathbf{D}_{\boldsymbol{\xi}}$ and $\mathbf{D}_{\boldsymbol{\Psi}}$ represent the corresponding diagonal matrices. It is clear that these matrices have strictly positive diagonal elements. From (8), it follows that

$$
\rho\left(\mathbf{D}_{\boldsymbol{\xi}} \mathbf{Z}\right)=\rho\left(\mathbf{D}_{\Psi} \mathbf{Z}\right)=1
$$

where $\rho(\mathbf{M})$ is spectral radius of matrix $\mathbf{M}$. For $\alpha \in(0,1)$, $\beta=1-\alpha$, let $\mathbf{D}_{\boldsymbol{\theta}}$ be the diagonal matrix corresponding to the point

$$
\boldsymbol{\theta}=\frac{1}{\rho\left(\alpha \mathbf{D}_{\boldsymbol{\xi}} \mathbf{Z}+\beta \mathbf{D}_{\Psi} \mathbf{Z}\right)}(\alpha \boldsymbol{\xi}+\beta \mathbf{\Psi}) .
$$

It follows from definition that

$$
\mathbf{D}_{\boldsymbol{\theta}}=\frac{1}{\rho\left(\alpha \mathbf{D}_{\boldsymbol{\xi}} \mathbf{Z}+\beta \mathbf{D}_{\Psi} \mathbf{Z}\right)}\left(\alpha \mathbf{D}_{\boldsymbol{\xi}}+\beta \mathbf{D}_{\Psi}\right) .
$$

Since the spectral radius of $\mathbf{D}_{\boldsymbol{\theta}} \mathbf{Z}$ is exactly 1 , from the Perron-Frobenius Theorem, (8) has a strictly positive solution for $\boldsymbol{\Gamma}=\boldsymbol{\theta}$ and, hence, $\boldsymbol{\theta}$ is in $S(\mathbf{Z})$. Due to the structure of the feasible region, we obtain the following criterion that the point $\alpha \boldsymbol{\xi}+\beta \Psi$ lies outside of the feasible region if and only if

$$
\rho\left(\alpha \mathbf{D}_{\boldsymbol{\xi}} \mathbf{Z}+\beta \mathbf{D}_{\Psi} \mathbf{Z}\right)>1
$$

or equivalently

$$
\rho\left(\alpha \mathbf{D}_{\boldsymbol{\xi}} \mathbf{Z}+\beta \mathbf{D}_{\Psi} \mathbf{Z}\right)>\alpha \rho\left(\mathbf{D}_{\boldsymbol{\xi}} \mathbf{Z}\right)+\beta \rho\left(\mathbf{D}_{\Psi} \mathbf{Z}\right) .
$$

A mathematical tool of central importance in this paper is the following two-variable polynomial:

$$
p(x, y)=\operatorname{det}\left(y\left(\mathbf{D}_{\boldsymbol{\xi}} \mathbf{Z}-\mathbf{I}\right)+\mathbf{D}_{\mathbf{\Psi}} \mathbf{Z}-x \mathbf{I}\right)
$$

which was adopted from [10] and was originally used to prove a theorem of Lax in regard to concavity of the eigenvalue function. The real roots of this polynomial are elements $(x, y)$ in $\mathbb{R}^{2}$ such that $p(x, y)=0$. We will show that the location of certain root branch of $p$ implies concavity. Some salient basic features of these real roots are summarized in the following lemma.

Lemma 1: For any $x$ there are at most $N-1$ real roots of the form $(x, y)$. For any non-negative $y$ there is at least one real 
root $(x, y)$ with $x>0$. In particular, the point $(1,0)$ is a root of $p$ and there is no root of the form $(x, 0)$ for $x>1$.

Proof: The coefficient of $y^{N}$ in $p(x, y)$ is $\operatorname{det}\left(\mathbf{D}_{\boldsymbol{\xi}} \mathbf{Z}-\mathbf{I}\right)$ which is equal to zero since $\boldsymbol{\xi}$ is in $S(\mathbf{Z})$. So the first statement holds. For any non-negative $y$, it follows that

$$
\rho\left(y \mathbf{D}_{\boldsymbol{\xi}} \mathbf{Z}+\mathbf{D}_{\mathbf{\Psi}} \mathbf{Z}\right)>\rho\left(y \mathbf{D}_{\boldsymbol{\xi}} \mathbf{Z}\right)=y .
$$

Note that the inequality in (15) is based on a well-known property of irreducible matrices (see, for example, [12]). Hence, there is a real root, $(x, y)$, for $p$ with

$$
\begin{aligned}
x & =\rho\left(y\left(\mathbf{D}_{\boldsymbol{\xi}} \mathbf{Z}-\mathbf{I}\right)+\mathbf{D}_{\Psi} \mathbf{Z}\right) \\
& =\rho\left(y \mathbf{D}_{\xi} \mathbf{Z}+\mathbf{D}_{\Psi} \mathbf{Z}\right)-y>0 .
\end{aligned}
$$

The last proposition statement follows from (9).

For any non-negative $y$, define

$$
m(y)=\rho\left(y \mathbf{D}_{\boldsymbol{\xi}} \mathbf{Z}+\mathbf{D}_{\boldsymbol{\Psi}} \mathbf{Z}\right)-y .
$$

The previous argument shows that $(m(y), y)$ defines a branch of real roots of $p$ and that for any non-negative $y, p$ has no other real root, $(x, y)$, such that

$$
x>m(y) .
$$

That is, $(m(y), y)$ is the rightmost root branch for non-negative $y$ (see Fig. 1 for an illustration). Note also that $m(y)$ is continuous in $y$. The usefulness of $m(y)$ in proofing concavity result is reflected by the following lemma.

Lemma 2: Let $\alpha, \beta>0$, then

$$
\begin{aligned}
\rho\left(\alpha \mathbf{D}_{\boldsymbol{\xi}} \mathbf{Z}+\beta \mathbf{D}_{\Psi} \mathbf{Z}\right)< & \alpha \rho\left(\mathbf{D}_{\boldsymbol{\xi}} \mathbf{Z}\right)+\beta \rho\left(\mathbf{D}_{\Psi} \mathbf{Z}\right) \\
& \text { if } m\left(\frac{\alpha}{\beta}\right)<1 \\
\rho\left(\alpha \mathbf{D}_{\boldsymbol{\xi}} \mathbf{Z}+\beta \mathbf{D}_{\Psi} \mathbf{Z}\right)> & \alpha \rho\left(\mathbf{D}_{\boldsymbol{\xi}} \mathbf{Z}\right)+\beta \rho\left(\mathbf{D}_{\Psi} \mathbf{Z}\right) \\
& \text { if } m\left(\frac{\alpha}{\beta}\right)>1 .
\end{aligned}
$$

Proof: Since $\rho\left(\mathbf{D}_{\boldsymbol{\xi}} \mathbf{Z}\right)=\rho\left(\mathbf{D}_{\Psi} \mathbf{Z}\right)=1$, it follows from (17) that for any $y>0$

$$
\begin{gathered}
\rho\left(y \mathbf{D}_{\boldsymbol{\xi}} \mathbf{Z}+\mathbf{D}_{\Psi} \mathbf{Z}\right)<y \rho\left(\mathbf{D}_{\boldsymbol{\xi}} \mathbf{Z}\right)+\rho\left(\mathbf{D}_{\Psi} \mathbf{Z}\right) \\
\text { if } m(y)<1 \\
\rho\left(y \mathbf{D}_{\boldsymbol{\xi}} \mathbf{Z}+\mathbf{D}_{\Psi} \mathbf{Z}\right)>y \rho\left(\mathbf{D}_{\boldsymbol{\xi}} \mathbf{Z}\right)+\rho\left(\mathbf{D}_{\Psi} \mathbf{Z}\right) \\
\text { if } m(y)>1 .
\end{gathered}
$$

The lemma follows by letting $y=\alpha / \beta$ and multiplying both sides of the inequality in (20) by $\beta$.

Lemma 3: Consider a symmetric $\mathbf{S}$ and a diagonal matrix $\mathbf{E}$ with strictly positive diagonal elements. The matrices $\mathbf{S}$ and $\mathbf{E S}$ have the same number of strictly positive (and strictly negative) eigenvalues.

Proof: Since $\operatorname{det} \mathbf{E} \neq 0$, the matrices $\mathbf{E S}$ and $\mathbf{E}^{1 / 2} \mathbf{S E} \mathbf{E}^{1 / 2}$ have the same characteristics equation, and, hence, they have the same set of eigenvalues counting algebraic multiplicities. On the other hand, by the Law of Inertia for quadratic forms [13] the number of strictly positive eigenvalues for $\mathbf{S}$ and $\mathbf{E}^{1 / 2} \mathbf{S} \mathbf{E}^{1 / 2}$ is identical. Similar result holds for strictly negative eigenvalues.

Theorem 4: Suppose $\mathbf{Z}$ is of the form $\mathbf{E S}$ where $\mathbf{E}$ is a diagonal matrix and $\mathbf{S}$ is symmetric. If $\mathbf{Z}$ is nonsingular and has only one strictly positive eigenvalue, then for any points $\boldsymbol{\xi}$ and $\boldsymbol{\Psi}$ in the boundary, $S(\mathbf{Z})$, the polynomial in $y, \operatorname{det}\left(y\left(\mathbf{D}_{\xi} \mathbf{Z}-\right.\right.$ I) $\left.+\mathbf{D}_{\Psi} \mathbf{Z}-\mathbf{I}\right)$, has only nonpositive real roots. Moreover, the feasible $\mathbf{S N R}$ region of $\mathbf{Z}$ is concave.

Proof: Define $m(y)$ as in (17). We will show that $m(y)>1$ for all strictly positive $y$.

Let $\mathbf{Y}=\mathbf{D}_{\mathbf{\Psi}}^{1 / 2} \mathbf{E}^{1 / 2} \mathbf{S} \mathbf{E}^{1 / 2} \mathbf{D}_{\mathbf{\Psi}}^{1 / 2}$. By the Law of Inertia for quadratic forms, the matrices $\mathbf{S}, \mathbf{E}^{1 / 2} \mathbf{S E}^{1 / 2}$ and $\mathbf{Y}$ have the same number of strictly positive (and strictly negative) eigenvalues. Since $\mathbf{E}^{1 / 2} \mathbf{S E}^{1 / 2}$ and $\mathbf{E S}=\mathbf{Z}$ have the same characteristic equation, it follows that $\mathbf{Y}$ has one strictly positive eigenvalue and $N-1$ strictly negative eigenvalues. Let $\mathbf{D}=$ $\mathbf{D}_{\boldsymbol{\Psi}} \mathbf{D}_{\boldsymbol{\xi}}^{-1}$. Define the polynomial $p(x, y)$ as in (14). We can regard $p$ as a polynomial in $y$ parametrized by $x$ and denote it by

$$
\begin{aligned}
q_{x}(y)= & \operatorname{det}\left(y\left(\mathbf{D}_{\boldsymbol{\xi}} \mathbf{Z}-\mathbf{I}\right)+\mathbf{D}_{\mathbf{\Psi}} \mathbf{Z}-x \mathbf{I}\right) \\
= & \operatorname{det}\left(y\left(\mathbf{D}^{-1} \mathbf{D}_{\mathbf{\Psi}}^{1 / 2} \mathbf{E}^{1 / 2} \mathbf{S E}^{1 / 2} \mathbf{D}_{\mathbf{\Psi}}^{1 / 2}-\mathbf{I}\right)\right. \\
& \left.+\mathbf{D}_{\mathbf{\Psi}}^{1 / 2} \mathbf{E}^{1 / 2} \mathbf{S} \mathbf{E}^{1 / 2} \mathbf{D}_{\mathbf{\Psi}}^{1 / 2}-x \mathbf{I}\right) \\
= & \operatorname{det}\left(y\left(\mathbf{D}^{-1} \mathbf{Y}-\mathbf{I}\right)+\mathbf{Y}-x \mathbf{I}\right) \\
= & \operatorname{det}\left(y\left(\mathbf{D}^{-1}-\mathbf{Y}^{-1}\right)+\mathbf{I}-x \mathbf{Y}^{-1}\right) \operatorname{det} \mathbf{Y} .
\end{aligned}
$$

Since $\mathbf{Y}$ and $\mathbf{D}_{\boldsymbol{\Psi}} \mathbf{E S}=\mathbf{D}_{\boldsymbol{\Psi}} \mathbf{Z}$ have the same set of eigenvalues, the positive eigenvalue of $\mathbf{Y}$ is 1 . If $0 \leq \varepsilon<1, \mathbf{I}-\varepsilon \mathbf{Y}^{-1}$ is a positive definite matrix. By the well-known result on positive definite pencil of symmetric matrices, there exists a real congruent transformation that puts $\mathbf{I}-\varepsilon \mathbf{Y}^{-1}$ and $\mathbf{D}^{-1}-\mathbf{Y}^{-1}$ into diagonal form simultaneously. In particular, the diagonal form of $\mathbf{I}-\varepsilon \mathbf{Y}^{-1}$ can be assumed to the identity matrix. By using the Law of Inertia one can show that the number of strictly negative roots of $q_{\varepsilon}(y)$ is equal to the number of strictly positive eigenvalues of $\mathbf{D}^{-1}-\mathbf{Y}^{-1}$ and in turn by Lemma 3 , is equal to the number of strictly positive eigenvalues of $\mathbf{I}-\mathbf{D Y} \mathbf{Y}^{-1}$, or equivalently $\mathbf{I}-\mathbf{D}^{1 / 2} \mathbf{Y}^{-1} \mathbf{D}^{1 / 2}$. Note that:

$$
\mathbf{D}^{1 / 2} \mathbf{Y}^{-1} \mathbf{D}^{1 / 2}=\mathbf{D}_{\boldsymbol{\xi}}^{-1 / 2} \mathbf{E}^{-1 / 2} \mathbf{S}^{-1} \mathbf{E}^{-1 / 2} \mathbf{D}_{\boldsymbol{\xi}}^{-1 / 2} .
$$

So $\mathbf{D}^{1 / 2} \mathbf{Y}^{-1} \mathbf{D}^{1 / 2}$, like $\mathbf{E}^{-1 / 2} \mathbf{S}^{-1} \mathbf{E}^{-1 / 2}$, has one strictly positive eigenvalue and $N-1$ strictly negative eigenvalues. Moreover, $\quad \mathbf{D}_{\xi}^{-1 / 2} \mathbf{E}^{-1 / 2} \mathbf{S}^{-1} \mathbf{E}^{-1 / 2} \mathbf{D}_{\xi}^{-1 / 2}$ and $\mathbf{Z}^{-1} \mathbf{D}_{\xi}^{-1}$ have the same set of eigenvalues which include the value $1=\rho\left(\mathbf{Z}^{-1} \mathbf{D}_{\boldsymbol{\xi}}^{-1}\right)=1 / \rho\left(\mathbf{D}_{\boldsymbol{\xi}} \mathbf{Z}\right)$. Thus, $\mathbf{I}-\mathbf{D}^{1 / 2} \mathbf{Y}^{-1} \mathbf{D}^{1 / 2}$ has $N-1$ strictly positive eigenvalue and a single eigenvalue at 0 . It follows that $q_{\varepsilon}(y)$ has $N-1$ strictly negative roots. As $\varepsilon$ tends to 1 , by continuity of roots to the characteristic equation, we conclude that there are no strictly positive roots for $q_{1}(y)$ ( 0 is always a root for $q_{1}(y)$ ). Now consider the function $m(y)$ defined in (17). Clearly, $m(0)=1$, which corresponds to the fact that $(1,0)$ is a real root for $q_{x}(y)$. For small enough strictly positive $y, m(y)$ must be greater than 1 , due to the fact that 


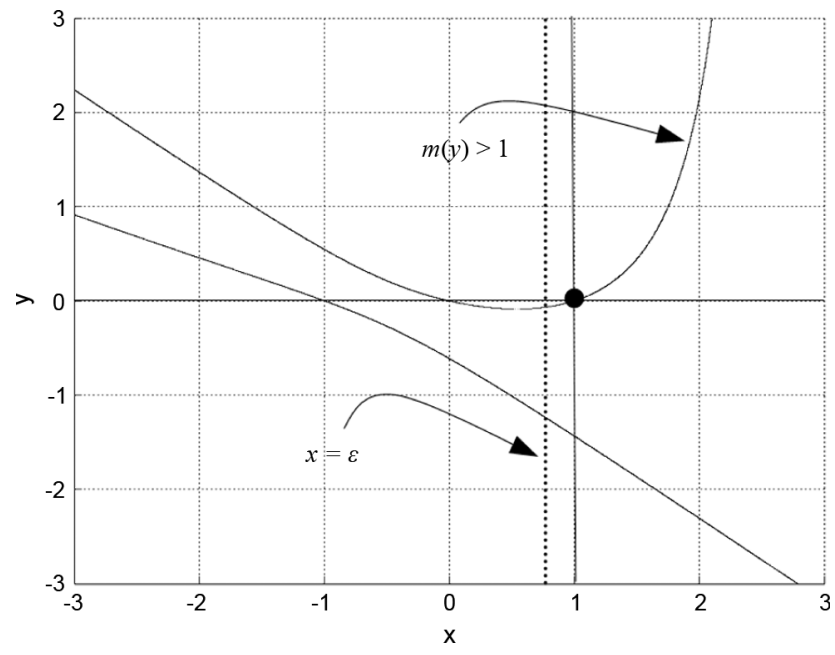

Fig. 1. $m(y)>1$ implies concavity. $q_{\epsilon}(y)$ has no strictly positive root.

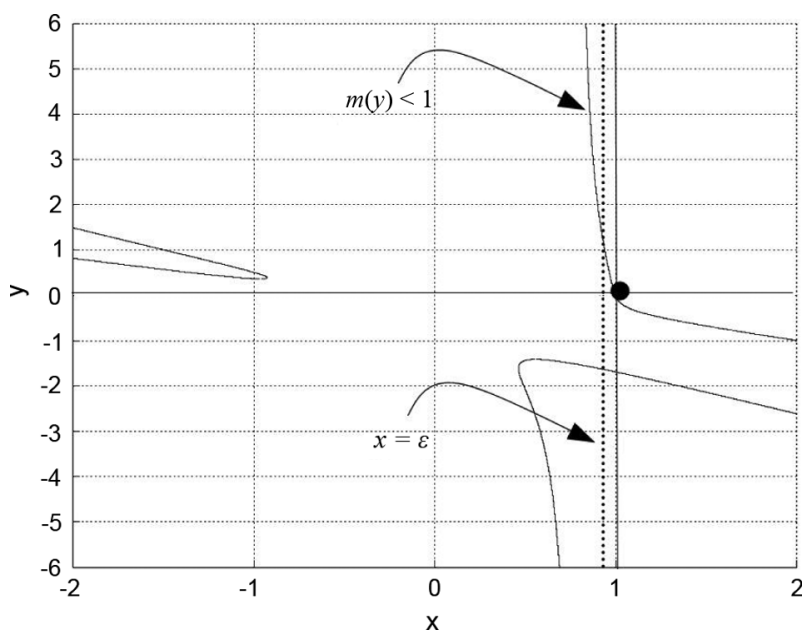

Fig. 2. $m(y)<1$ implies convexity. $q_{\epsilon}(y)$ has a strictly positive root.

$q_{x}(y)$ cannot have a strictly positive root for $x \in[0,1]$ (see Figs. 1 and 2 for an illustration). Thus, $m(y)>1$ holds for all positive $y$ in a small enough neighborhood around 0 . As $y$ increases, the continuous curve $(m(y), y)$ cannot cross the line $x=1$ as $q_{1}(y)$ has no strictly positive roots. By Lemma 2 and using the condition defined in (13), it follows that the feasible region is concave.

In Section $\mathrm{V}$ we will show by numerical examples that if $\mathbf{Z}$ has more than one strictly positive eigenvalue, the feasible SNR region is not concave even if $\mathbf{Z}$ is symmetric. Since $\mathbf{D}_{\boldsymbol{\xi}} \mathbf{Z}$ is primitive, it has a simple root at 1 , so there exists a nonsingular matrix, $\mathbf{Q}$, such that

$$
\mathbf{D}_{\boldsymbol{\xi}} \mathbf{Z}=\mathbf{Q}\left(\begin{array}{cc}
\mathbf{1} & \mathbf{0}_{N-1}^{T} \\
\mathbf{0}_{N-1} & \Lambda_{0}
\end{array}\right) \mathbf{Q}^{-1}
$$

where $\boldsymbol{\Lambda}_{0}$ is an $(N-1) \times(N-1)$ sub-matrix and $\mathbf{0}_{N-1}$ and $\mathbf{0}_{N-1}^{T}$ are $(N-1) \times 1$ and $1 \times(N-1)$ zero matrices respectively. It follows from Perron-Frobenious Theorem that $\operatorname{det}\left(\boldsymbol{\Lambda}_{0}-\mathbf{I}_{N-1}\right) \neq 0$ and $\operatorname{sgn} \operatorname{det}\left(\boldsymbol{\Lambda}_{0}-\mathbf{I}_{N-1}\right)=(-1)^{N-1}$. If we write

$$
\mathbf{Q}=\left[\mathbf{p}, \mathbf{R}_{0}\right], \quad \mathbf{Q}^{-1}=\left[\begin{array}{l}
\mathbf{q}^{T} \\
\mathbf{S}_{0}
\end{array}\right]
$$

then it follows directly from (23) that

$$
\mathbf{D}_{\boldsymbol{\xi}} \mathbf{Z} \mathbf{p}=\mathbf{p}, \quad \mathbf{q}^{T} \mathbf{D}_{\boldsymbol{\xi}} \mathbf{Z}=\mathbf{q}^{T}, \quad \mathbf{q}^{T} \mathbf{p}=1 .
$$

It follows that

$$
\mathrm{p} \gg 0, \quad \mathrm{q} \gg 0 .
$$

Recall that $\mathbf{D}=\mathbf{D}_{\boldsymbol{\Psi}} \mathbf{D}_{\boldsymbol{\xi}}^{-1}$, hence

$$
\mathbf{q}^{T} \mathbf{D p}>0 .
$$

Lemma 5: Under the previous notation

$$
\begin{aligned}
& p(x, y)=\operatorname{det}\left(y\left(\mathbf{D}_{\boldsymbol{\xi}} \mathbf{Z}-\mathbf{I}\right)+\mathbf{D}_{\mathbf{\Psi}} \mathbf{Z}-x \mathbf{I}\right)= \\
& \operatorname{det}\left[\begin{array}{cc}
\mathbf{q}^{T} \mathbf{D} \mathbf{p}-x & \mathbf{q}^{T} \mathbf{D} \mathbf{R}_{0} \boldsymbol{\Lambda}_{0} \\
\mathbf{S}_{0} \mathbf{D p} & y\left(\boldsymbol{\Lambda}_{0}-\mathbf{I}_{N-1}\right)-x \mathbf{I}_{N-1}+\mathbf{S}_{0} \mathbf{D} \mathbf{R}_{0} \mathbf{\Lambda}_{0}
\end{array}\right] .
\end{aligned}
$$

This result can also be verified by direct computation and details are omitted here.

Proposition 6: Consider a general primitive interference matrix, Z. If the polynomial in $y$

$$
\begin{aligned}
q_{1}(y) & =\operatorname{det}\left(y\left(\mathbf{D}_{\xi} \mathbf{Z}-\mathbf{I}\right)+\mathbf{D}_{\mathbf{\Psi}} \mathbf{Z}-\mathbf{I}\right) \\
& =c_{N-1} y^{N-1}+\cdots+c_{1} y
\end{aligned}
$$

has only nonpositive roots, then for all strictly positive $y$, the property $m(y)>1$ holds if and only if

$$
c_{N-1}(-1)^{N-1}>0 .
$$

Moreover, (30) holds if and only if

$$
c_{1}(-1)^{N-1}>0 .
$$

Proof: Let $\varepsilon$ be an arbitrarily small but fixed strictly positive number. Let

$$
x_{-}=\mathbf{q}^{T} \mathbf{D} \mathbf{p}-\varepsilon, \quad x_{+}=\mathbf{q}^{T} \mathbf{D} \mathbf{p}+\varepsilon .
$$

If $x$ is equal to $x_{-}$or $x_{+}$, then for all large enough $y$ the dominant term in the determinant in (28) is

$$
\left(\mathbf{q}^{T} \mathbf{D} \mathbf{p}-x\right) \operatorname{det}\left(\boldsymbol{\Lambda}_{0}-\mathbf{I}_{N-1}\right) y^{N-1}
$$

and the sign of $p(x, y)$ is equal to the sign of the expression in (33). It follows that for all large enough $y, p(x, y)$ changes sign as $x$ varies from $x_{-}$to $x_{+}$and has a real root between $x_{-}$and 
$x_{+}$. By continuity of the roots, as $\varepsilon$ tends to zero, there is a root branch that tends to $\left(\mathbf{q}^{T} \mathbf{D} \mathbf{p}, \infty\right)$. On the other hand, since

$$
p(x, y)=y^{N} \operatorname{det}\left(\left(\mathbf{D}_{\boldsymbol{\xi}} \mathbf{Z}-\mathbf{I}\right)+\frac{1}{y} \mathbf{D}_{\Psi} \mathbf{Z}-\frac{x}{y} \mathbf{I}\right),
$$

for a fixed, large enough positive $y$, there is a one-to-one relation between $x$ roots of $p(x, y)$ with strictly negative real parts and eigenvalues of $\mathbf{D}_{\boldsymbol{\xi}} \mathbf{Z}-\mathbf{I}$ with strictly negative real parts. There are $N-1$ such eigenvalues of $\mathbf{D}_{\boldsymbol{\xi}} \mathbf{Z}-\mathbf{I}$. We have also shown that for large enough positive $y, p(x, y)$ has one strictly positive real root in $x$ in a neighborhood of $\mathbf{q}^{T} \mathbf{D p}$. Hence, this strictly positive root is unique and must be part of the root branch defined by $(m(y), y)$. As the neighborhood can be made arbitrarily small as $y$ approaches infinity, for large enough positive $y, m(y)>1$ if and only if $\mathbf{q}^{T} \mathbf{D} \mathbf{p}>1$. By proposition assumption, $q_{1}(y)$ has no strictly positive root so $(m(y), y)$ cannot cross the vertical line $x=1$. Hence, $m(y)>1$ holds all strictly positive $y$ if and only if it holds for some large enough positive $y$.

On the other hand, from (28) and (29) it follows that

$$
c_{N-1}=\left(\mathbf{q}^{T} \mathbf{D} \mathbf{p}-1\right) \operatorname{det}\left(\boldsymbol{\Lambda}_{0}-\mathbf{I}_{N-1}\right) .
$$

Therefore, for all $y>0, m(y)>1$ if and only if $c_{N-1}(-1)^{N-1}>0$. To prove the last proposition statement, note that

$$
\begin{aligned}
q_{1}(y) & =y^{N} q_{1}(1 / y) \\
& =y^{N} \operatorname{det}\left(\mathbf{D}_{\boldsymbol{\xi}} \mathbf{Z}-\mathbf{I}+\frac{1}{y}\left(\mathbf{D}_{\Psi} \mathbf{Z}-\mathbf{I}\right)\right) \\
& =y^{N}\left(\frac{c_{N-1}}{y}+\cdots+\frac{c_{1}}{y^{N-1}}\right) .
\end{aligned}
$$

Hence, $c_{1}$ is the coefficient of the leading term of the following polynomial in $z$

$$
\operatorname{det}\left(\mathbf{D}_{\xi} \mathbf{Z}-\mathbf{I}+z\left(\mathbf{D}_{\Psi} \mathbf{Z}-\mathbf{I}\right)\right) .
$$

One can repeat previous arguments by reversing the role of $\boldsymbol{\xi}$ and $\Psi$. In particular, one can define

$$
n(z)=\rho\left(\mathbf{D}_{\boldsymbol{\xi}} \mathbf{Z}+z \mathbf{D}_{\Psi} \mathbf{Z}\right)-z
$$

as in (17) and show that $n(z)>1$ for all $z>0$ if and only if $c_{1}(-1)^{N-1}>0$. By setting $z=1 / y$, one can check that $m(y)>1$ for all $y>0$ if and only if $n(z)>1$ for all $z>0$. The last statement of the proposition follows.

\section{CONCAVITY THEOREM FOR 3-D SYSTEMS}

When $N=2$, the feasible SNR region is concave, that is, the infeasible region in $\mathbb{R}_{+}^{2}$ is convex [5], [6]. For $N \geq 4$, the concavity result has been shown in [9] to be invalid in general. In this paper, concavity of the feasible SNR region is established for all 3-D systems. Consider first of all the case that $\mathbf{Z}$ is symmetric. Since $\mathbf{Z}$ has all diagonal elements equal to 0 this implies the sum of its eigenvalues is 0 . Other than the dominant eigenvalue, the other two eigenvalues must be strictly negative, since each one must have absolute value strictly less than the dominant eigenvalue. Theorem 4 then implies that for all 3-D systems with symmetric primitive interference matrix $\mathbf{Z}$ the feasible SNR region is concave.

Theorem 7: The feasible SNR region of a 3-D power control system with a primitive interference matrix $\mathbf{Z}$ is concave.

Before proving this result, we need to establish the following lemma for 3-D systems.

Lemma 8: For any 3-D primitive interference matrix, $\mathbf{Z}$, the polynomial in $y$

$$
q(y)=\operatorname{det}\left(y\left(\mathbf{D}_{\boldsymbol{\xi}} \mathbf{Z}-\mathbf{I}\right)+\mathbf{D}_{\Psi} \mathbf{Z}-\mathbf{I}\right)
$$

has a strictly negative root in addition to the root at 0 .

Proof: Let

$$
\begin{aligned}
\mathbf{D}_{\boldsymbol{\xi}} \mathbf{Z} & =\left(\begin{array}{lll}
0 & a & b \\
c & 0 & d \\
e & f & 0
\end{array}\right) \\
\mathbf{D} & =\mathbf{D}_{\boldsymbol{\Psi}} \mathbf{D}_{\boldsymbol{\xi}}^{-1}=\left(\begin{array}{ccc}
d_{1} & 0 & 0 \\
0 & d_{2} & 0 \\
0 & 0 & d_{3}
\end{array}\right) .
\end{aligned}
$$

If $\mathbf{D}_{\xi} \mathbf{Z}$ has two complex roots, clearly $\operatorname{det} \mathbf{D}_{\xi} \mathbf{Z}>0$. Otherwise, two of the three real roots must be strictly negative which also implies that $\operatorname{det} \mathbf{D}_{\boldsymbol{\xi}} \mathbf{Z}>0$.

Note that the $d_{i}$ 's are strictly positive. If one of the $d_{i}$ 's is equal to 1 , then one can show by direct verification that $y=-1$ is a root to $q(y)$, proving the lemma. On the other hand, results from Perron-Frobenius theory implies it is impossible that all $d_{i}$ 's are less than 1 . Thus, without loss of generality, we can assume that

$$
d_{1} \leq d_{2}<1<d_{3}
$$

(If not, we can reverse the role of $\boldsymbol{\xi}$ and $\boldsymbol{\Psi}$ by considering $y^{3} q(1 / y)$, which has the same number of strictly negative roots as $q(y))$.

Then

$$
\begin{aligned}
q(-1) & =\operatorname{det}\left(\mathbf{D}_{\mathbf{\Psi}} \mathbf{Z}-\mathbf{D}_{\boldsymbol{\xi}} \mathbf{Z}\right) \\
& =\left(d_{1}-1\right)\left(d_{2}-1\right)\left(d_{3}-1\right) \operatorname{det} \mathbf{D}_{\boldsymbol{\xi}} \mathbf{Z}>0 .
\end{aligned}
$$

On the other hand

$$
q\left(-d_{2}\right)=\left(d_{2}-1\right)^{3}-\left(d_{2}-1\right)\left(d_{1}-d_{2}\right)\left(d_{3}-d_{2}\right) b e<0 .
$$

So $q(y)$ has a root in the interval $\left[-1,-d_{2}\right]$.

Proof of Theorem 7: Construct a continuous one-parameter family of matrices by

$$
\mathbf{Z}_{r}=\mathbf{Z}+r \mathbf{Z}^{T} .
$$

Define a family of characteristic polynomials

$$
\begin{aligned}
q_{r}(y) & =\operatorname{det}\left(y\left(\frac{\mathbf{D}_{\boldsymbol{\xi}} \mathbf{Z}_{r}}{\rho\left(\mathbf{D}_{\boldsymbol{\xi}} \mathbf{Z}_{r}\right)}-\mathbf{I}\right)+\frac{\mathbf{D}_{\boldsymbol{\Psi}} \mathbf{Z}_{r}}{\rho\left(\mathbf{D}_{\boldsymbol{\Psi}} \mathbf{Z}_{r}\right)}-\mathbf{I}\right) \\
& =a_{r} y^{2}+b_{r} y .
\end{aligned}
$$

The coefficient functions, $a_{r}$ and $b_{r}$, are continuous. From the proof of Lemma 8 , one can see that these functions cannot 


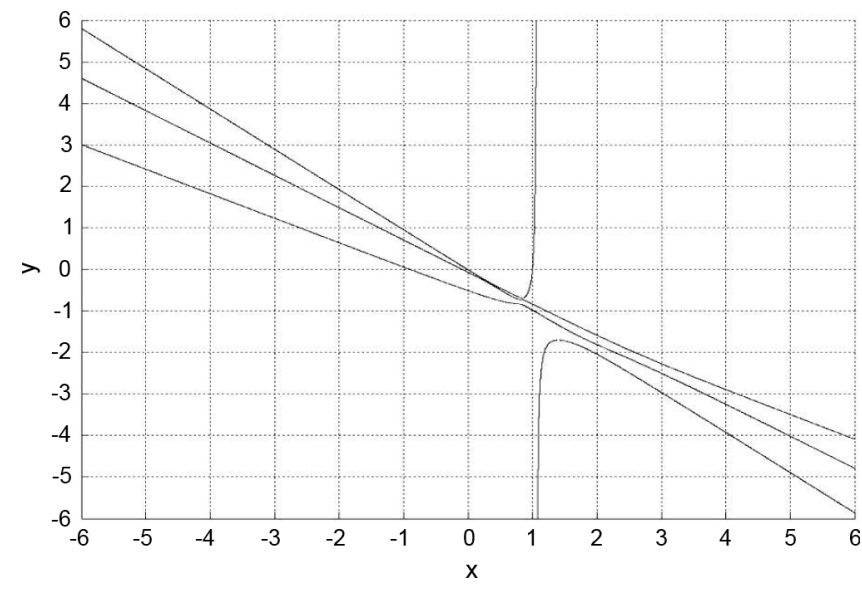

Fig. 3. A 4-D symmetric example showing concavity property.

vanish since any $q_{r}(y)$ has a root at zero and a strictly negative root. Thus

$$
\operatorname{sgn}\left(a_{0}\right)=\operatorname{sgn}\left(a_{1}\right)>0 .
$$

The last inequality follows from Lemma 2 and Proposition 6 since the feasible region of $\mathbf{Z}+\mathbf{Z}^{T}$ is concave. The feasible region for $\mathbf{Z}$ is, therefore, concave since $\operatorname{sgn}\left(a_{0}\right)>0$.

\section{NuMERICAL EXAMPLES}

In this section, we present some numerical examples to illustrate the results reported previously. First of all, we note a 4-D example which satisfies the eigenvalue condition stated in Theorem 4. Consider the following system:

$$
\hat{\mathbf{Z}}=\left(\begin{array}{cccc}
0 & 0.1 & 0.1 & 0.1 \\
0.1 & 0 & 0.2 & 0.1 \\
0.1 & 0.2 & 0 & 0.5 \\
0.1 & 0.1 & 0.5 & 0
\end{array}\right)
$$

The eigenvalues of $\hat{\mathbf{Z}}$ are

$$
(0.6235,-0.0126,-0.1000,-0.5110) \text {. }
$$

If

$$
\mathbf{E}_{1}=\left(\begin{array}{cccc}
1 & 0 & 0 & 0 \\
0 & 2 & 0 & 0 \\
0 & 0 & 3 & 0 \\
0 & 0 & 0 & 4
\end{array}\right), \quad \mathbf{E}_{2}=\left(\begin{array}{cccc}
4 & 0 & 0 & 0 \\
0 & 3 & 0 & 0 \\
0 & 0 & 2 & 0 \\
0 & 0 & 0 & 1
\end{array}\right)
$$

and $\mathbf{D}_{1}=\mathbf{E}_{1} / \rho\left(\mathbf{E}_{1} \hat{\mathbf{Z}}\right)$ and $\mathbf{D}_{2}=\mathbf{E}_{2} / \rho\left(\mathbf{E}_{2} \hat{\mathbf{Z}}\right)$, then Fig. 3 shows the roots of the polynomial

$$
p_{1}(x, y)=\operatorname{det}\left(y\left(\mathbf{D}_{2} \hat{\mathbf{Z}}-\mathbf{I}_{4}\right)+\mathbf{D}_{1} \hat{\mathbf{Z}}-x \mathbf{I}_{4}\right) .
$$

If we redefine $\mathbf{E}_{1}$ and $\mathbf{E}_{2}$ so that

$$
\mathbf{E}_{1}=\left(\begin{array}{cccc}
.1 & 0 & 0 & 0 \\
0 & 3 & 0 & 0 \\
0 & 0 & 3 & 0 \\
0 & 0 & 0 & 1
\end{array}\right), \quad \mathbf{E}_{2}=\left(\begin{array}{cccc}
20 & 0 & 0 & 0 \\
0 & .1 & 0 & 0 \\
0 & 0 & 2 & 0 \\
0 & 0 & 0 & 4
\end{array}\right)
$$

then the real roots of $p_{1}$ are shown in Fig. 4.

We also consider one case where $\mathbf{Z}$ is a nonsymmetric matrix with only one eigenvalue with strictly positive real part. Let

$$
\hat{\mathbf{Z}}=\left(\begin{array}{cccc}
0 & 0.11 & 0.3 & 0.3 \\
0.2 & 0 & 0.2 & 0.1 \\
0.1 & 0.2 & 0 & 0.7 \\
0.1 & 0.2 & 0.3 & 0
\end{array}\right)
$$

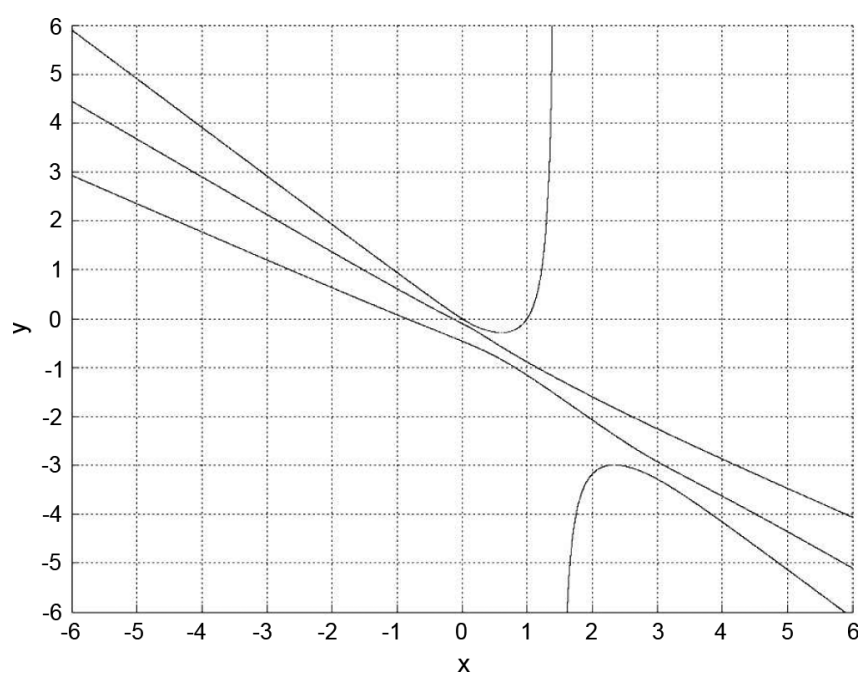

Fig. 4. A 4-D symmetric example showing concavity property.

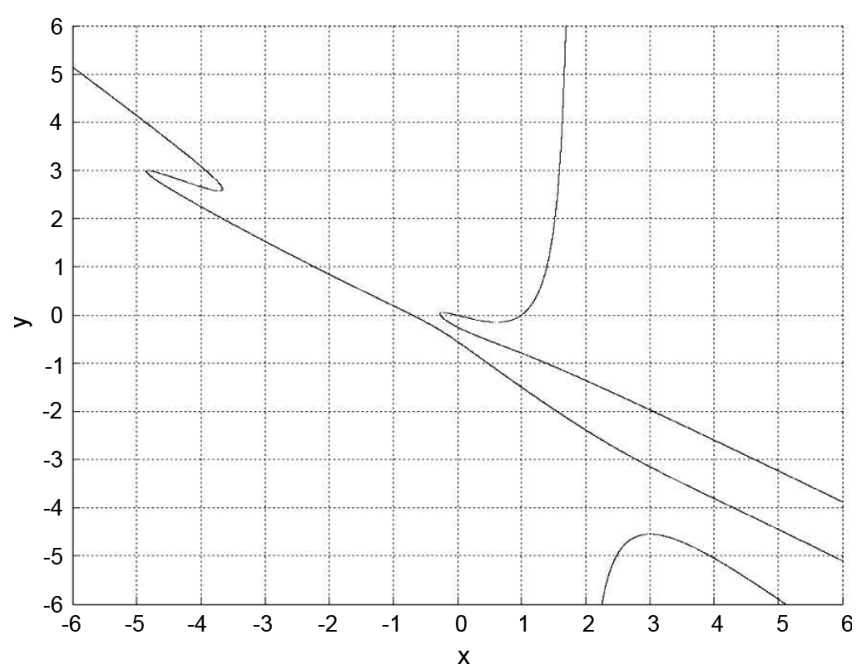

Fig. 5. A 4-D nonsymmetric example showing concavity property.

The eigenvalues are

$(0.7069,-0.1345+0.1086 i,-0.1345-0.1086 i,-0.4379)$.

For $\mathbf{E}_{1}$ and $\mathbf{E}_{2}$ defined as in (50), the real roots of $p_{1}$ are shown in Fig. 5.

The assumption of a single strictly positive eigenvalue is a key assumption for concavity. Consider the following symmetric case with

$$
\hat{\mathbf{Z}}=\left(\begin{array}{cccc}
0 & 0.2 & 0.01 & 0.02 \\
0.2 & 0 & 0.2 & 0.01 \\
0.01 & 0.2 & 0 & 0.3 \\
0.02 & 0.01 & 0.3 & 0
\end{array}\right)
$$

The eigenvalues are $(0.3952,0.1363,-0.1534,-0.3781)$. Define $\mathbf{E}_{1}$ and $\mathbf{E}_{2}$ as in (48), then the real roots of $p_{1}$ are shown in Fig. 6. 


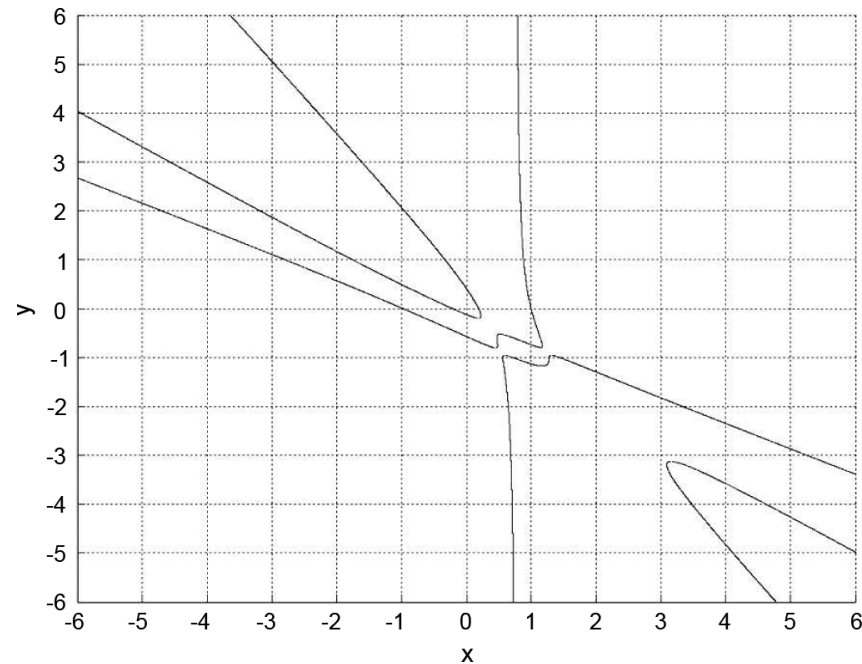

Fig. 6. A 4-D symmetric example showing concavity does not hold.

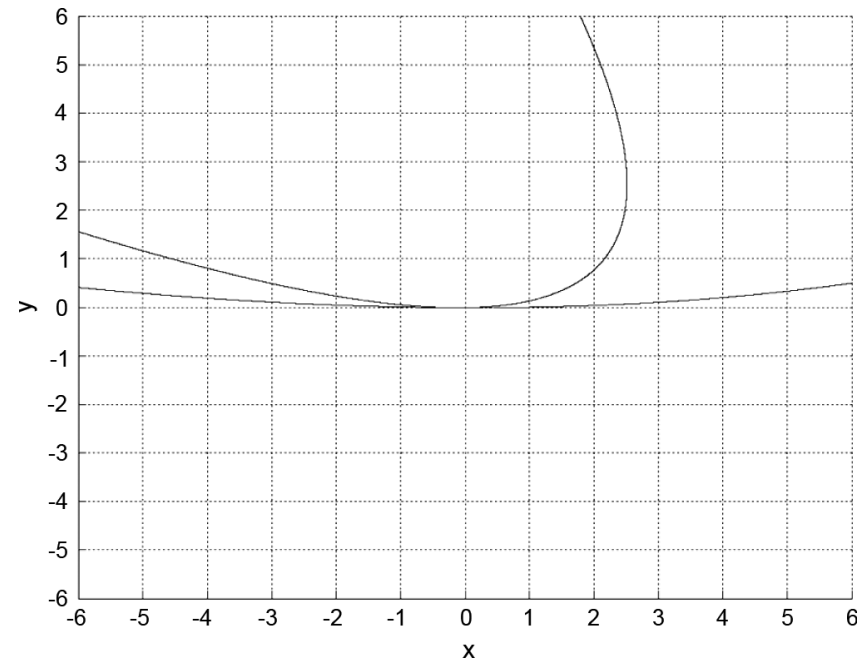

Fig. 7. Same example showing concavity property.

However, when one sets

$$
\begin{aligned}
\mathbf{E}_{1} & =\left(\begin{array}{cccc}
.1 & 0 & 0 & 0 \\
0 & 10 & 0 & 0 \\
0 & 0 & .1 & 0 \\
0 & 0 & 0 & 10
\end{array}\right) \\
\mathbf{E}_{2} & =\left(\begin{array}{cccc}
10 & 0 & 0 & 0 \\
0 & .1 & 0 & 0 \\
0 & 0 & 10 & 0 \\
0 & 0 & 0 & .1
\end{array}\right)
\end{aligned}
$$

then the line segment joining the corresponding points on the boundary of the feasible region exhibits a concavity property as shown in Fig. 7 (the curve $(m(y), y)$ runs close to the $x$-axis).

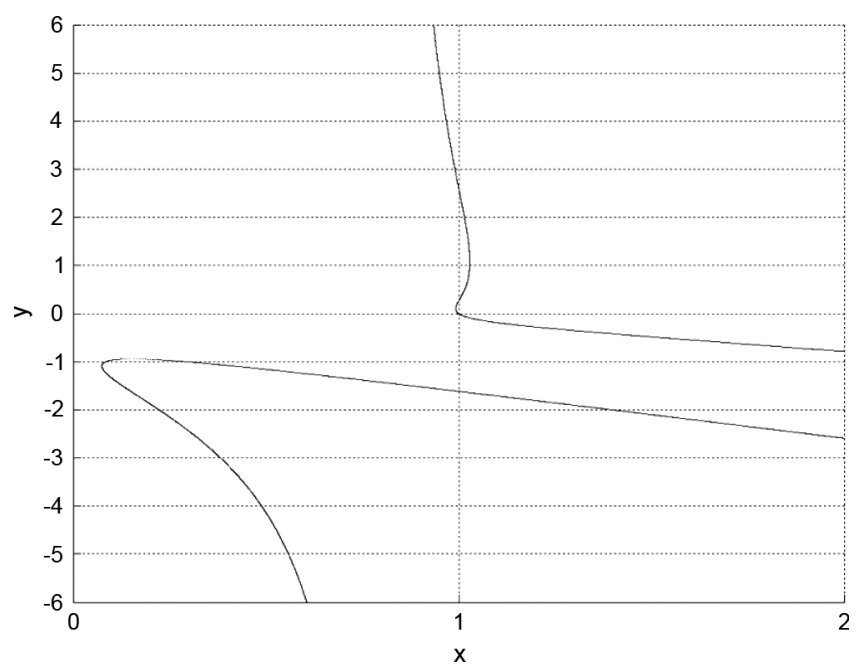

Fig. 8. Example showing the complexity of the feasible region geometry.

In fact, it is noted that the concavity property can be made very drastic. If we label the two feasible points defined in (53) as $\boldsymbol{\xi}$ and $\bar{\Psi}$, with

$$
\begin{aligned}
\boldsymbol{\xi} & =[0.227,22.729,0.227,22.729]^{T} \\
\bar{\Psi} & =[23.236,0.232,23.236,0.232]^{T}
\end{aligned}
$$

then the feasible point in the direction defined by the vector $0.5 * \boldsymbol{\xi}+0.5 * \overline{\boldsymbol{\Psi}}$ is

$$
[2.558,2.558,2.558,2.558]^{T}
$$

The SNR of some of the users are reduced by a factor close to 10.

Finally, we note with one example that the geometry of the feasible SNR region can be very complex. Consider a 5-D system with

$$
\begin{aligned}
\hat{\mathbf{Z}} & =\left(\begin{array}{ccccc}
0 & 0.8 & 0.4 & 0.008 & 0.08 \\
0.08 & 0 & 0.8 & 0.004 & 0.008 \\
0.004 & 0.08 & 0 & 0.08 & 0.04 \\
0.08 & 0.004 & 0.08 & 0 & 0.08 \\
0.16 & 0.008 & 0.4 & 0.08 & 0
\end{array}\right) \\
\mathbf{E}_{1} & =\left(\begin{array}{ccccc}
0.01 & 0 & 0 & 0 & 0 \\
0 & 0.01 & 0 & 0 & 0 \\
0 & 0 & 3 & 0 & 0 \\
0 & 0 & 0 & 5 & 0 \\
0 & 0 & 0 & 0 & 5
\end{array}\right) \\
\mathbf{E}_{2} & =\left(\begin{array}{ccccc}
5 & 0 & 0 & 0 & 0 \\
0 & 5 & 0 & 0 & 0 \\
0 & 0 & 0.03 & 0 & 0 \\
0 & 0 & 0 & 0.01 & 0 \\
0 & 0 & 0 & 0 & 0.01
\end{array}\right) .
\end{aligned}
$$

Then for $\mathbf{D}_{1}=\mathbf{E}_{1} / \rho\left(\mathbf{E}_{1} \hat{\mathbf{Z}}\right)$ and $\mathbf{D}_{2}=\mathbf{E}_{2} / \rho\left(\mathbf{E}_{2} \hat{\mathbf{Z}}\right)$, the real roots of

$$
p_{2}(x, y)=\operatorname{det}\left(y\left(\mathbf{D}_{2} \hat{\mathbf{Z}}-\mathbf{I}_{5}\right)+\mathbf{D}_{1} \hat{\mathbf{Z}}-x \mathbf{I}_{5}\right)
$$


is shown in Fig. 8. On close examination, one can see the line joining the corresponding feasible points on the boundary is divided into three segments. The middle segment is outside the feasible region while the remaining two segments are inside the feasible region. So neither concavity nor convexity holds even for the lining joining these two points.

\section{CONCLUSION}

In this paper, concavity results of the feasible signal-to-noise regions are established. The results shed light on the complexity of the geometric properties of these feasible regions. There are several possible directions for future extension. In particular, in Theorem 4 , the symmetry and the nonsingular assumptions may not be necessary.

\section{ACKNOWLEDGMENT}

The author would like to thank Dr. H. Cheng for helping with the MATLAB programming used in this paper. It is the belief of the author that this paper would not have been completed without the help of MATLAB.

\section{REFERENCES}

[1] M. Sunay and P. McLane, "Calculating error probabilities for DS-CDMA systems: When not to use the Gaussian approximation," in Proc. IEEE GLOBECOM, London, U.K., 1996, pp. 1744-1749.

[2] Y. Chen and W. S. Wong, "Power control for non-Gaussian interference," in Proc. WiOpt'09, Seoul, Korea, 2009, pp. 161-168.

[3] C. W. Sung, "Log-convexity property of the feasible SIR region in power-controlled cellular systems," IEEE Commun. Lett., vol. 6, pp. 248-249, 2002.
[4] D. Catrein, L. A. Imhof, and R. Mathar, "Power control, capacity and duality of uplink and downlink in cellular CDMA systems," IEEE Trans. Commun., vol. 52, pp. 1777-1785, Oct. 2004.

[5] H. Boche and S. Stanczak, "Convexity of some feasible QoS regions and asymptotic behavior of the minimum total power in CDMA systems," IEEE Trans. Commun., vol. 52, pp. 2190-2197, Dec. 2004.

[6] H. Boche and S. Stanczak, "Log-convexity of the minimum total power in CDMA systems with certain quality-of-service guaranteed," IEEE Trans. Inf. Theory, vol. 51, no. 1, pp. 374-381, Jan. 2005.

[7] L. A. Imhof and R. Mathar, "The geometry of the capacity region for CDMA systems with general power constraints," IEEE Trans. Wireless Commun., vol. 4, pp. 2040-2044, Sep. 2005.

[8] L. A. Imhof and R. Mathar, "Capacity regions and optimal power allocation for CDMA cellular radio," IEEE Trans. Inf. Theory, vol. 51, no. 6, pp. 2011-2019, Jun. 2005.

[9] S. Stanczak and H. Boche, "The infeasible SIR region is not a convex set," IEEE Trans. Commun., vol. 54, pp. 1905-1907, 2006.

[10] H. F. Weinberger, "Remarks on the preceding of Lax," Commun. Pure Appl. Math., vol. 11, pp. 195-196, 1958.

[11] P. D. Lax, "Differential equations, difference equations and matrix theory," Commun. Pure Appl. Math., vol. 11, pp. 175-194, 1958.

[12] A. Berman and R. J. Plemmons, Nonnegative Matrices in the Mathematical Sciences. New York: Academic, 1994.

[13] F. R. Gantmacher, Matrix Theory. Providence, RI: American Mathematical Society, 2000, vol. I.

Wing Shing Wong (M'81-SM'90-F'02) received the combined B.S. and M.S. degree (summa cum laude) from Yale University, New Haven, CT, in 1976, and the M.S. and Ph.D. degrees from Harvard University, Cambridge, MA, in 1978 and 1980 , respectively.

After working at AT\&T Bell Laboratories, Holmdel, NJ, for ten years, in 1992, he joined the Chinese University of Hong Kong, Shatin, and is now a Professor of information engineering. He was the Chairman of the Department of Information Engineering from 1995 to 2003 and is currently serving as the Dean of the Graduate School. He served as the Science Advisor at the Innovation and Technology Commission of the HKSAR Government from 2003 to 2005. He has participated in a variety of research areas including mobile communication systems, nonlinear filtering, search engine and estimation, and control of finite communication bandwidth systems.

Prof. Wong is a Co-Editor-in-Chief of Communications in Information and Systems. 